OPEN ACCESS

Edited by:

Mingqiang Li,

Sun Yat-sen University, China

Reviewed by:

Hanze Hu,

Columbia University, United States

Lusha Tong,

Zhejiang University, China

Gaiqing Wang,

Shanxi Medical University, China

*Correspondence:

Lisha Li

lilisha@jlu.edu.cn

Yulin Li

ylli@jlu.edu.cn

Specialty section:

This article was submitted to Biomaterials,

a section of the journal

Frontiers in Bioengineering and

Biotechnology

Received: 29 April 2020

Accepted: 22 June 2020

Published: 14 July 2020

Citation:

Sun $Y, X u Z$, Wang $M, L v S$, Wu H, Chi G, Li L and Li Y (2020) Soft

Matrix Combined With BMPR Inhibition Regulates Neurogenic Differentiation of Human Umbilical Cord Mesenchymal Stem Cells.

Front. Bioeng. Biotechnol. 8:791.

doi: 10.3389/fbioe.2020.00791

\section{Soft Matrix Combined With BMPR Inhibition Regulates Neurogenic Differentiation of Human Umbilical Cord Mesenchymal Stem Cells}

\author{
Yingying Sun ${ }^{1,2}$, Ziran $X u^{1}$, Meijing Wang ${ }^{1}$, Shuang $L v^{1}$, Haitao Wu ${ }^{1}$, Guangfan Chi ${ }^{1}$, \\ Lisha $\mathrm{Li}^{* *}$ and Yulin $\mathrm{Li}^{{ }^{*}}$ \\ ${ }^{1}$ The Key Laboratory of Pathobiology, Ministry of Education, College of Basic Medical Sciences, Jilin University, Changchun, \\ China, ${ }^{2}$ Department of Stomatology, First Hospital of Jilin University, Changchun, China
}

Stem cells constantly encounter as well as respond to a variety of signals in their microenvironment. Although the role of biochemical factors has always been emphasized, the significance of biophysical signals has not been studied until recently. Additionally, biophysical elements, like extracellular matrix (ECM) stiffness, can regulate functions of stem cells. In this study, we demonstrated that soft matrix with 1-10 kPa can induce neural differentiation of human umbilical cord mesenchymal stem cells (hUCMSCs). Importantly, we used a combination of soft matrix and bone morphogenetic protein receptor (BMPR) inhibition to promote neurogenic differentiation of hUCMSCs. Furthermore, BMPR/SMADs occurs in crosstalk with the integrin $\beta 1$ downstream signaling pathway. In addition, BMPR inhibition plays a positive role in maintaining the undifferentiated state of hUC-MSCs on the hydrogel substrate. The results provide further evidence for the molecular mechanisms via which stem cells convert mechanical inputs into fateful decisions.

\footnotetext{
Keywords: stiffness, neural differentiation, bone morphogenetic protein receptor, mesenchymal stem cells, hydrogel
}

\section{INTRODUCTION}

Various types of stem cells and progenitor cells have the ability to demonstrate the stiffness of extracellular matrix (ECM; Smith et al., 2018). The cytoskeleton, morphology, and migration of these cells all respond to ECM mechanics within a few hours, and then influence cell proliferation, and/or differentiation at following days (Rammensee et al., 2017). It is important that phenotypic changes in cell morphology occur with several hours: myoblast-like elongation on an intermediate stiff gel, osteoblasts expand on a stiff gel, and neuron-like dendritic branching on a soft gel (Lv et al., 2015).

Discher et al. indicated that the culture of mesenchymal stem cells (MSCs) on a very soft surface enhanced neuromorphological changes and the expression of neural genes (Engler et al., 2006). In particular, MSCs implanted in the polyacrylamide gel with a surface modulus of $<1 \mathrm{kPa}$, suggesting the stiffness of brain tissues, highly expression of neural markers, such as neural structural proteins, including neurofilament and $\beta$-III tubulin, and microtubule-associated protein 2 (Hadden et al., 2017). Moreover, other studies have demonstrated that the soft surfaces have a neural-inducing 
effect on some types of stem cells such as induced pluripotent stem cells, adult neural stem cells, and epidermal stem cells (Thompson and Chan, 2016). There are four major types of polymers that are used in mechano-sensitivity studies: polyacrylamide (PAAM), polydimethylsiloxane (PDMS), and polyelectrolyte multilayer films made of synthetic polyelectrolytes, which are mostly employed as 2D culture substrates; whereas the fourth, poly (ethylene glycol; PEG), is used as a 3D hydrogel with cells embedded in it (Her et al., 2013). Some researchers have synthesized type I collagen and hyaluronic acid scaffolds, found that MSCs were likely to differentiate into neuronal lineage in substrate of $1 \mathrm{kPa}$, while transformed into glial cells in matrix of $10 \mathrm{kPa}$ (Her et al., 2013). Soft matrix has been displayed to increase the neuronal function and neurogenic differentiation (Lv et al., 2015; Mao et al., 2016).

Stem cells are extremely sensitive to their physical environment, but it is not clear how physical stimuli signals are transduced in the cells (Stukel and Willits, 2018). It has been reported that the BMP signaling pathway is a crucial pathway associated with neural induction of stem cells on stiff surfaces, tissue development, and cell differentiation (Thompson and Chan, 2016). Sun et al. substantiated the inhibitory effect of soft poly (dimethylsiloxane) on Smad1/5/8 phosphorylation of human pluripotent stem cells using immunoblots technique. Indeed, it is supported that YAP/TAZ-mediated nuclear accumulation of SMADs regulates stiffness-dependent neural induction of stem cells (Dupont et al., 2011; Sun et al., 2014). Moreover, in these different cellular responses, focal adhesions formed by integrin clustering are considered to modulate several pathways, making them an vital component of substrate-mediated transduction (Stukel and Willits, 2016).

After understanding the process of interaction between human umbilical cord mesenchymal stem cells (hUC-MSCs) and their physical environment, it is possible to control the differentiation direction of hUC-MSCs by the construction of substrates with unique stiffness characteristics. According to our previous work, PAAM prepared by polymerizing cross-linking of acrylamide and bis-acrylamide, are being used to construct substrates of 1-10, 35-38, and 62$68 \mathrm{kPa}$. In this study, we adopted a method that soft matrix combined with small molecule inhibitor to regulate the neurogenic differentiation of hUC-MSCs, and to investigate the molecular mechanism underlying how biophysical signals are transduced.

\section{RESULTS}

\section{Matrix Stiffness Regulates the Morphology and Neural Differentiation}

The morphological characteristics of hUC-MSCs changed dramatically when cultured on matrix gels with different stiffness at days 1 and 7 . From the results, comparing day 1 with the control group (cells on Tissue Culture Plate TCP), cells on matrix with $1-10 \mathrm{kPa}$ became two ends shortened and round; cells on $35-38 \mathrm{kPa}$ showed spindleshaped; while cells on $62-68 \mathrm{kPa}$ were well spread and irregularly shaped, with a few cells still being spindle-shaped. Furthermore, the morphological differences in cells in each group at day 7 were more apparent compared to those at day 1 (Figure 1B).

Subsequently, the aspect ratios (ratio of cell length to width) of hUC-MSCs at day 1 and day 7 were calculated. It is obvious that the aspect ratio in the stiffness groups was significantly lower than that in the control group, at both day 1 and day 7 . In the stiffness groups, the length-width ratios of hUC-MSCs were highest on $35-38 \mathrm{kPa}$, followed by $62-68 \mathrm{kPa}$, and the lowest on 1-10 $\mathrm{kPa}$. Under the same culture conditions, the cell morphology changed markedly in each stiffness group, which indicated that matrix stiffness affected the morphology of the cells. In addition, there was no significant difference in aspect ratios over time in each group (Figure 1C). Then the cell area of hUC-MSCs was measured at days 1 and 7. The results showed that, at day 1 , the area of cells on $1-10 \mathrm{kPa}$ was the smallest, medium on $35-38 \mathrm{kPa}$, and the largest on $62-68 \mathrm{kPa}$. Interestingly, the spreading area of these cells was increased with the increasing stiffness. However, at day 7, the spreading area of cells on $62-68 \mathrm{kPa}$ decreased instead. In general, the spreading ability of the cells was higher on $35-38 \mathrm{kPa}$ than that on $1-10 \mathrm{kPa}$ soft matrix and $62-68 \mathrm{kPa}$ stiff matrix. The results showed that the cell area increased gradually on $1-10 \mathrm{kPa}$ over time, but it was not the same on $62-68 \mathrm{kPa}$, which may be related to cell differentiation (Figure 1D).

Thereafter, the neural stem cell marker (Nestin) and the specific marker in early stage of neurons ( $\beta$ III-tubulin) were detected using qRT-PCR. It was shown that the expression levels of Nestin and BIII-tubulin were higher in hUC-MSCs on 1-10 kPa than TCP and the other two groups (35$38 \mathrm{kPa}$ and $62-68 \mathrm{kPa}$ ). With the stiffness increasing, the expression of neural markers decreased (Figure 1E). It confirms that hUC-MSCs highly expressed neuronal markers on matrix of $1-10 \mathrm{kPa}$, that is stiffness of $1-10 \mathrm{kPa}$ induced the neural differentiation of hUC-MSCs. Subsequently, the total proteins of hUC-MSCs were collected at day 7, and western blotting was utilized to examine the protein expression levels of Nestin and $\beta$ III-tubulin. The results showed that, the cells highly expressed Nestin, and $\beta$ III-tubulin on $1-10 \mathrm{kPa}$ compared with the TCP group, which was consistent with the results of the qRT-PCR. It further proved that hUCMSCs tend to differentiate in neural direction on $1-10 \mathrm{kPa}$ (Figure 1F). However, the expression of $\beta$ III-tubulin on 35$38 \mathrm{kPa}$ was higher than that on $1-10 \mathrm{kPa}$, which conflicted with the result of mRNA detection, which may be affected by many other factors.

Furthermore, mRNA expression of stem cell self-renewal specific genes SOX2 and OCT4 were detected using qRTPCR. The results showed that SOX2 and OCT4 expression by hUC-MSCs on $1-10,35-38$, and $62-68 \mathrm{kPa}$ were prominently downregulated compared with that of TCP, as well as there was a difference between each group (Figure 1G). These genes included some that are known to participate in the process of self-renewal; those observed indicated that the 
A

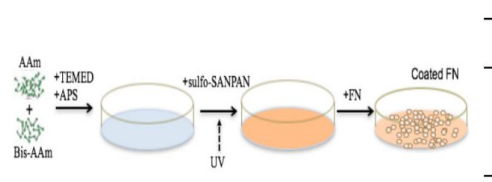

B
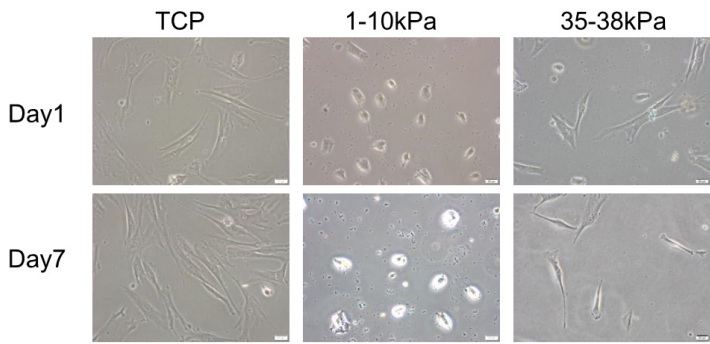

$62-68 \mathrm{kPa}$

E
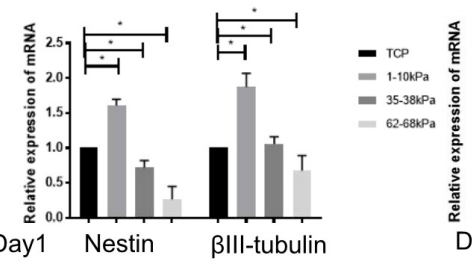

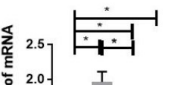

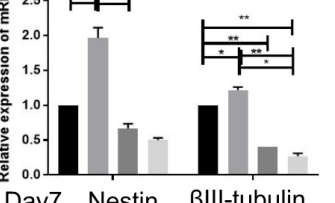

G

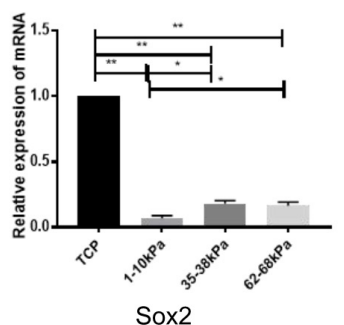

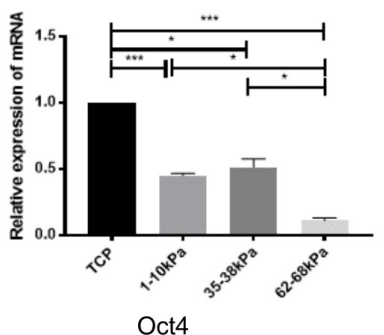

C

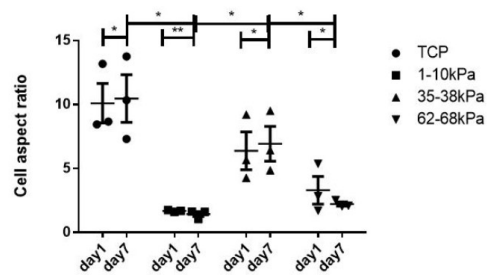

D

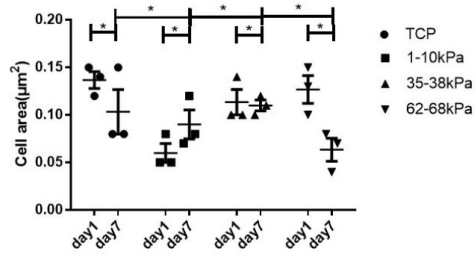

$\mathbf{F}$
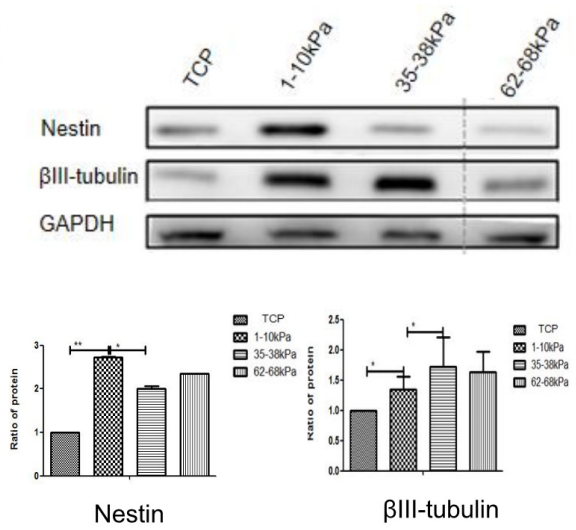

FIGURE 1 | Matrix stiffness regulates cell morphology and neural differentiation ofhuman umbilicalcordmesenchymal stem cells (hUC-MSCs). (A) A summary of substrate stiffness construction. Matrixes of different stiffness were prepared by mixing the same concentration of acrylamide (AAm) with different concentrations of bis-acrylamide (Bis-AAm; 0.05, 0.3, and 0.7\%). Sulfo-SANPAH (hexanoate) was cross-linked via ultraviolet irradiation and then coated with fibronectin for cell adhesion. (B) Matrix stiffness affects the morphological characteristics of hUC-MSCs. Morphological characteristics of hUC-MSCs were observed under a scanning electron microscope at days 1 and 7. TCP group: cells on Tissue Culture Plate. Scale bar $=20 \mu \mathrm{m}$. $n=3$ independent wells. (C) The cell aspect ratio was calculated using $\mathrm{NIH}$ Image J. The aspect ratio of the cell is the ratio of the major to minor axes $\left(n=3,{ }^{*} p<0.05\right.$, and $\left.{ }^{* *} p<0.01\right)$. (D) The cell area was calculated using $\mathrm{NIH}$ Image $\mathrm{J}\left(n=3,{ }^{*} p<0.05\right.$, and $\left.{ }^{* *} p<0.01\right)$. (E) qRT-PCR analyses were performed to detect the expression of neuronal-specific markers Nestin and $\beta$ III-tubulin in hUC-MSCs at day 1 and day 7 (Results are presented as mean \pm SEM, $n=5$ independent experiments, ${ }^{*} p<0.05$, and ${ }^{* *} p<0.01$ ). (F) Western blotting analysis of Nestin and $\beta$ III-tubulin at day 7 (Results are presented as mean \pm SEM, $n=3,{ }^{*} p<0.05$, and ${ }^{* *} p<0.01$ ). (G) qRT-PCR analysis of stem cells self-renewal markers SOX2 and OCT4 at day 1 (Results are presented as mean $\pm \mathrm{SEM}, n=5$ independent experiments, ${ }^{*} p<0.05,{ }^{* \star} p<0.01$, and ${ }^{* \star *} p<0.001$ ).

self-renewal ability of hUC-MSCs decreased, which was induced by stiffness of matrix.

\section{Soft Matrix Combined With BMPR Inhibition Enhances the Neural Differentiation of hUC-MSCs}

The mRNA expression of bone morphogenetic protein receptor (BMPR; BMPRIA, BMPRIB, and BMPRII) was detected using qRT-PCR after cells were cultured for $24 \mathrm{~h}$ and 7 days. The highest expression level of BMPR was found on matrix of 1-10 kPa. BMPRIA was 7.9-fold higher, BMPRIB was 8.5fold higher, and BMPRII was 7.5-fold higher than that of the control group. In addition, the expression of BMPR subtype decreased gradually with increasing stiffness, and there was a difference between each group (Figure 2A). Moreover, BMPR (BMPRIA, BMPRIB, and BMPRII) expression in the cells at the protein level was detected using western blotting at day 7 . Results showed that, the expression of BMPRIA in hUC-MSCs was significantly higher than that of the TCP group, with a statistically significant difference. However, the expression of BMPRII on 1-10 kPa was the lowest (Figure 2B). It has been reported that BMPRIA is involved in the neural differentiation which is regulated by stiffness, therefore we investigated the role of BMPRIA in the following experiments (Du et al., 2011; Xu et al., 2014).

To further explore the role of BMPR, we selected BMPR inhibitor (LDN-193189) to inhibit BMPR. The concentration gradient of BMPR inhibitor was $0,2,3,5$, 10 , and $15 \mu \mathrm{M}$ according to previous reports (Yu et al., 2008; 
A

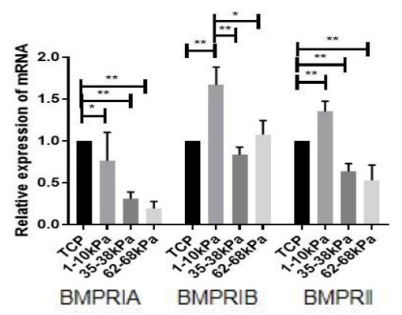

C

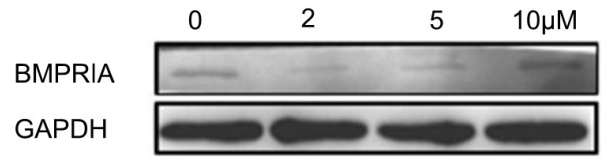

B

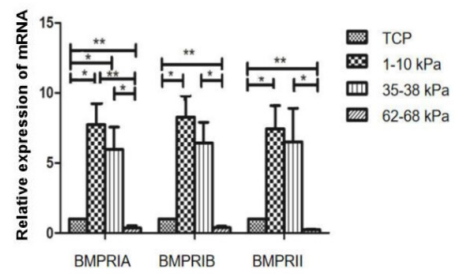

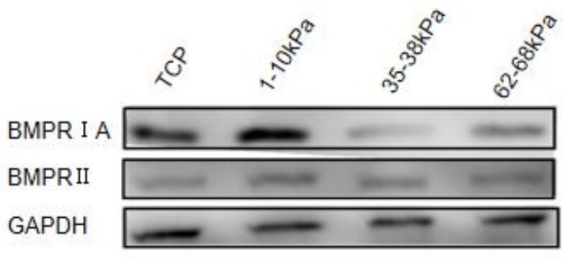

E

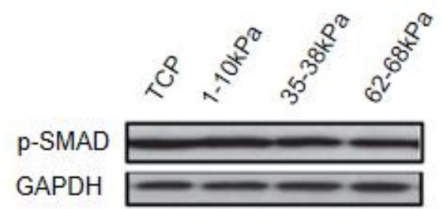

Inhibitor-

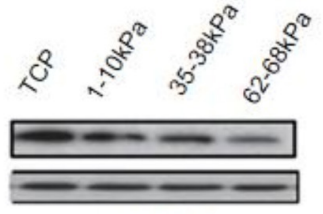

Inhibitor+

D
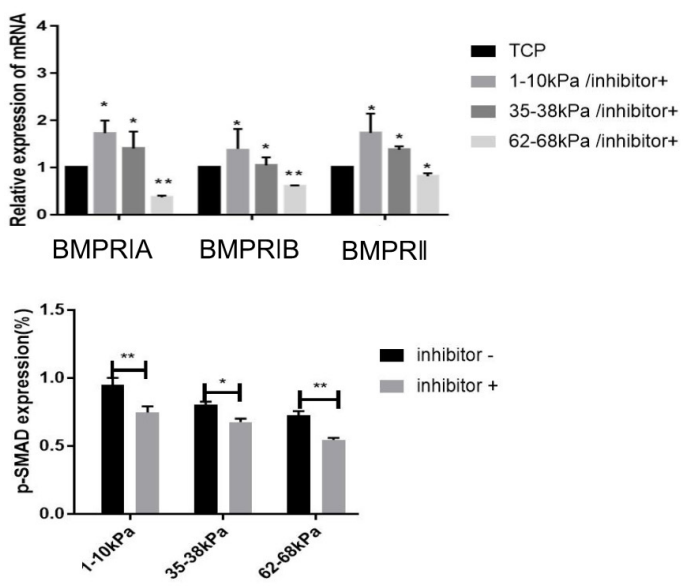

F

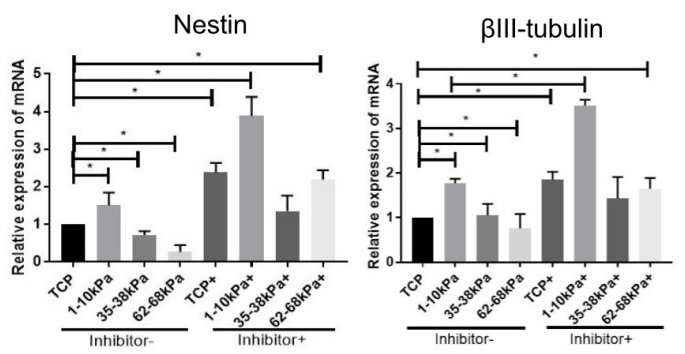

G

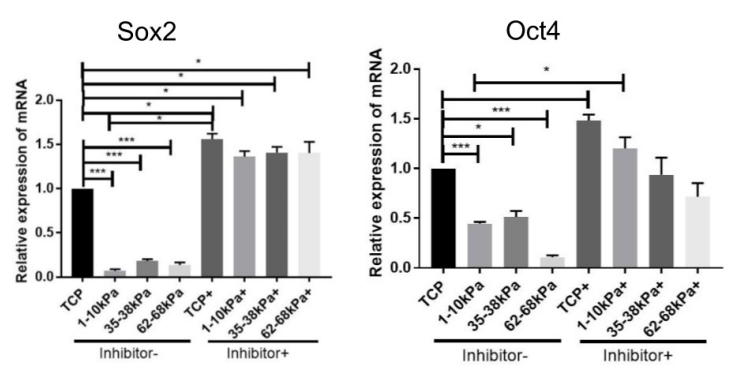

FIGURE 2 | BMPR is involved in stiffness-mediated neural differentiation of hUC-MSCs. (A) qRT-PCR detection of BMPR subtypes expression at day 1 and day 7 (Results are presented as mean \pm SEM, $n=5$ independent experiments, ${ }^{*} p<0.05$, and ${ }^{* *} p<0.01$ ). (B) Expression of BMPR subtypes detected using western blotting, and statistical analysis diagram (Results are presented as mean $\pm \mathrm{SEM}, n=3,{ }^{*} p<0.05$, and ${ }^{\star *} p<0.01$ ). (C) The best concentration of BMPR inhibitor was determined using western blotting, the best concentration of inhibitor was $2 \mu \mathrm{M}(n=3$ independent experiments). (D) The cells were collected at day 7 for detection of BMPR expression after stiffness groups adding inhibitor using RT-gPCR (Results are presented as mean $\pm \mathrm{SEM}, n=5$ independent experiments, ${ }^{*} p<0.05$, and ${ }^{* *} p<0.01$ ). (E) Expression of $p$-SMAD was detected using western blotting before and after BMPR inhibition, and statistical analysis diagram (Results are presented as mean \pm SEM, $n=3,{ }^{*} p<0.05$, and $\left.{ }^{* *} p<0.01\right)$. (F) qRT-PCR analysis of Nestin and $\beta$ Ill-tubulin expression after BMPR inhibition for $24 \mathrm{~h}$. Statistical analysis was performed using one-way ANOVA. Statistical significance was defined as $p<0.05$. Results are presented as mean \pm SEM $(n=5$ independent experiments,

$p<0.05)$. (G) qRT-PCR analysis of SOX2 and OCT4 expression after BMPR inhibition for $24 \mathrm{~h}$. Statistical analysis was performed using one-way ANOVA. Statistical significance was defined as $p<0.05$. Results are presented as mean $\pm \operatorname{SEM}\left(n=5\right.$ independent experiments, $p<0.05$, and $\left.{ }^{* \star *} p<0.001\right)$.

Al Madhoun et al., 2016). The best concentration of BMPR inhibitor was detected using western blotting. Under a microscope, the cell death rate was higher when the concentration of the inhibitor was 10 and $15 \mu \mathrm{M}$, therefore, the concentration of the inhibitor was $0,2,3$, and $5 \mu \mathrm{M}$ in this study. When the concentration of the inhibitor was $2 \mu \mathrm{M}$, BMPR expression level in hUC-MSCs was significantly inhibited compared with that of the non-inhibitor group (Figure 2C), thus the concentration of the inhibitor was $2 \mu \mathrm{M}$ in subsequent experiments. Next, BMPR expression in cells, after inhibition for 7 days, was detected using qRT-PCR. It was shown that after adding inhibitor, the expression of BMPR in hUC-MSCs on 1-10 $\mathrm{kPa}$ was still higher than that on TCP (the control group without inhibitor), but it was significantly lower than that without inhibitor at day 7, which proved that BMPR was effectively suppressed (Figure 2D). Furthermore, the expression of BMPR downstream protein p-SMAD was detected using western blotting after adding BMPR inhibitor for 7 days. We found that the expression of P-SMAD was downregulated in all groups after BMPR inhibition and decreased with increasing stiffness (Figure 2E).

Next, the expression levels of Nestin and $\beta$ III-tubulin in hUCMSCs after BMPR inhibition were detected. It is found that the expression of Nestin and $\beta$ III-tubulin were both significantly upregulated after BMPR inhibition in all groups (Figure 2F). Moreover, the expression of neural markers in 1-10 $\mathrm{kPa}$ was 
A

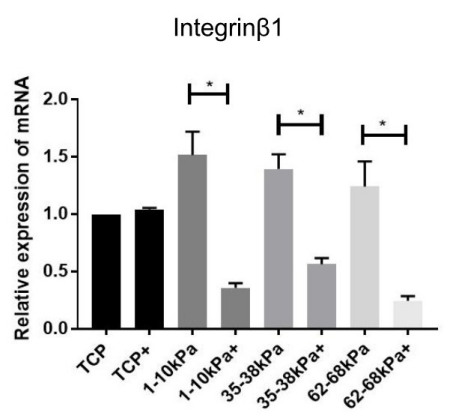

B

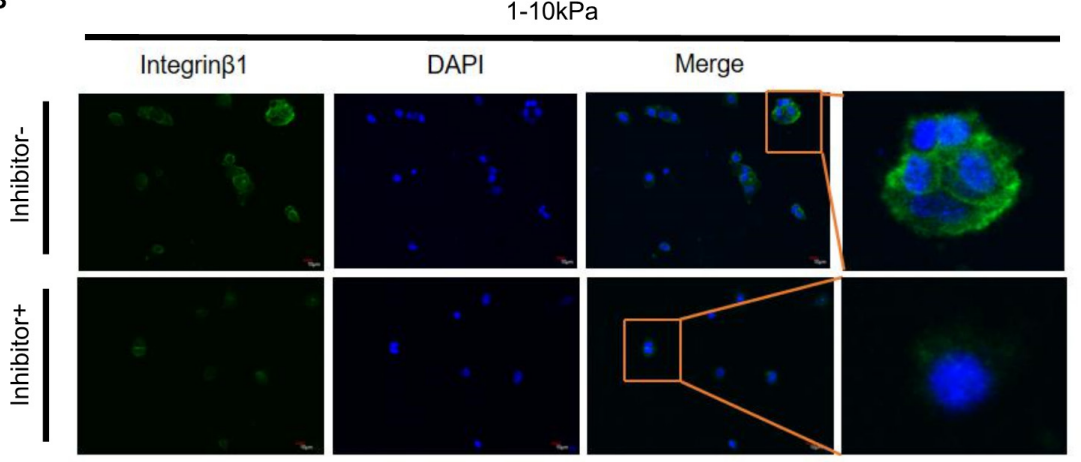

C

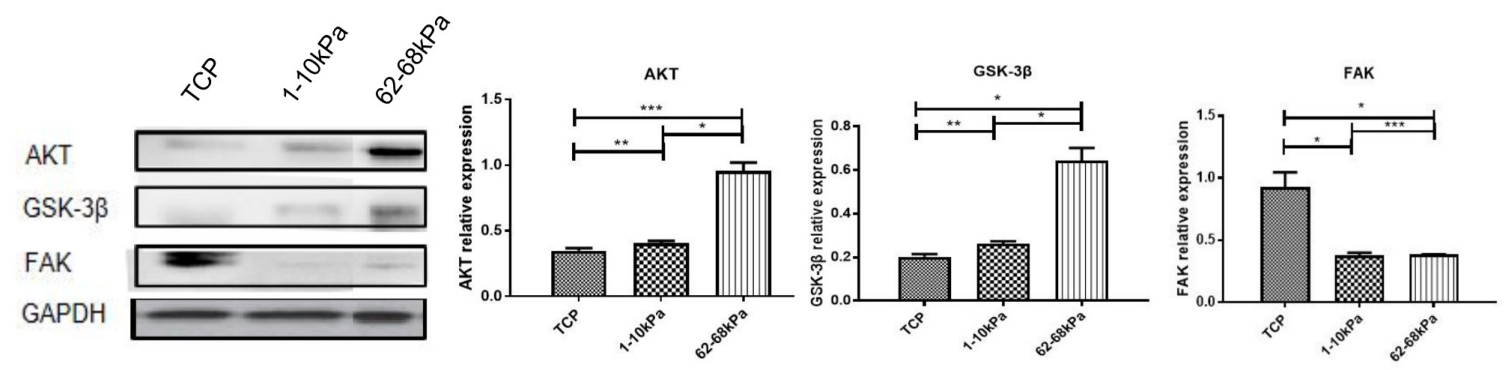

D

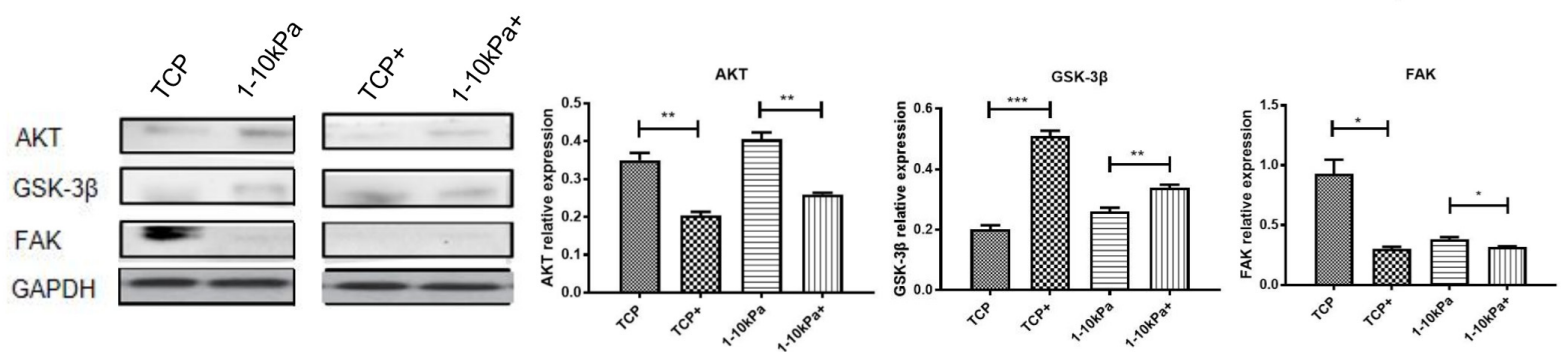

FIGURE 3 | The downstream molecules of integrin $\beta 1$ were detected after BMPR inhibition. (A) qRT-PCR analysis of integrin $\beta 1$ after BMPR inhibition for 24 h. Results are presented as mean \pm SEM $\left(n=5\right.$ independent experiments, ${ }^{*} p<0.05$, and $\left.{ }^{* *} p<0.01\right)$. "+"represents the group contained inhibitor. (B) Staining for integrin $\beta 1$ in hUC-MSCs, which were treated on 1-10 kPa before and after BMPR inhibition for $24 \mathrm{~h}$, and then observed under a confocal microscope (Olympus FV $1200 ; n=3$ independent experiments, scale bars, $10 \mu \mathrm{m})$. (C) Western blotting detection and statistical analysis diagram showing proteins associated with integrin $\beta 1$ including AKT, GSK-3 $\beta$, and FAK on TCP, $1-10 \mathrm{kPa}$, and 62-68 kPa before adding inhibitor (Results are presented as mean $\pm \mathrm{SEM}, n=3$, ${ }^{*} p<0.05$, ${ }^{* *} p<0.01$, and $\left.{ }^{* * *} p<0.001\right)$. (D) Western blotting detection of expression of AKT, GSK-3 $\beta$, and FAK on TCP and 1-10 kPa after adding inhibitor (Results are presented as mean \pm SEM, $n=3,{ }^{*} p<0.05,{ }^{* *} p<0.01$, and $\left.{ }^{* \star *} p<0.001\right)$. "+" represents the group contained inhibitor.

the highest in different stiffness groups after BMPR inhibition (Figure 2F). It proved that soft matrix of 1-10 kPa combined with BMPR inhibition can further enhance the ability of hUCMSCs neural differentiation. In addition, we detected the mRNA expression of stem cell self-renewal specific genes SOX2 and OCT4 after BMPR inhibition using qRT-PCR. It was shown that SOX2 and OCT4 expression in hUC-MSCs in all groups was prominently upregulated compared to those not inhibited, and there was a difference between each group (Figure 2G). It indicated that the self-renewal ability of hUC-MSCs on the hydrogel matrix was increased after BMPR inhibition. From these results, we conclude that the $1-10 \mathrm{kPa}$ soft matrix promotes the neurogenic differentiation of hUC-MSCs, and BMPR may play a negative role during this process. In addition, compared with the TCP group, the hydrogel stiffness regulates the directional differentiation of hUC-MSCs, and when BMPR is inhibited, the characteristics of hUC-MSCs are maintained and the differentiation is inhibited, indicating that BMPR has a positive regulatory effect on the characteristics of hUC-MSCs in each stiffness group.

\section{BMPR Has Crosstalk With Integrin Pathway in Soft Matrix Inducing Neural Differentiation of hUC-MSCs}

To detect the changes of integrin pathway in the process that soft matrix promotes the neural differentiation of hUCMSCs, we detected the mRNA expression of integrin $\beta 1$ after BMPR inhibition for $24 \mathrm{~h}$ using qRT-PCR. It was shown that, integrin $\beta 1$ expression in hUC-MSCs after BMPR inhibition in three stiffness groups decreased prominently compared with those not inhibited, and there was a difference between each group (Figure 3A). Then, we focused on 1-10 kPa and performed immunofluorescence staining of integrin $\beta 1$ 
at $24 \mathrm{~h}$. The results showed that integrin $\beta 1$ was mainly distributed on the cell membrane before BMPR inhibition. Moreover, integrin $\beta 1$ expression decreased significantly after BMPR inhibition (Figure 3B). In addition, total hUC-MSCs proteins were collected at day 7 , and the levels of integrin related proteins FAK, AKT, and GSK-3 $\beta$ were detected using western blotting. Comparing the three groups of TCP, $1-10 \mathrm{kPa}$ soft matrix and $62-68 \mathrm{kPa}$ stiff matrix, the protein expression levels of AKT and GSK-3 $\beta$ in the two stiffness groups were higher than those in the TCP, representing the original state of cells, and increased with increasing stiffness; while the level of FAK in the stiffness groups was lower than that in the TCP, but there was no significant difference between the stiffness groups (Figure 3C). This indicates that at this point of time, AKT and GSK-3 $\beta$ were involved in stiffness regulating the directional differentiation of hUC-MSCs, whereas FAK did not play an important role at this time. Next, we compared the levels of integrin related signaling proteins before and after BMPR inhibition in the TCP and 1$10 \mathrm{kPa}$ groups. The results showed that in the TCP group, the levels of AKT and FAK decreased after BMPR inhibition, while the level of GSK-3 $\beta$ increased (Figure 3D). It indicates that BMPR inhibitor decreased the levels of AKT and FAK but suppressed the level of GSK-3 $\beta$ in the primitive state of hUCMSCs. In the $1-10 \mathrm{kPa}$ group, after adding BMPR inhibitor, the level of AKT was downregulated and GSK-3 $\beta$ was upregulated, while the level of FAK showed no significant change (Figure 3D). This suggests that AKT and GSK-3 $\beta$ cooperated with BMPR to be involved in the soft matrix promoting neural differentiation of hUC-MSCs, while FAK was not involved in this regulatory process at this point.

\section{DISCUSSION}

In recent years, increasing attention has been given to the application of hUC-MSCs in cell therapy (Shang et al., 2017). Three properties make MSCs optimal for tissue regeneration:immunoregulatory capacity beneficial to alleviate abnormal immune responses; paracrine or autocrine functions that generate growth factors, and the ability to differentiate into target cells (Han et al., 2019). This study adopted a new method to promote neural differentiation of hUC-MSCs in vitro and laid a solid theoretical foundation for the design of regenerated materials in tissue engineering. In this study, polyacrylamide hydrogel was successfully constructed to imitate the stiffness of brain and bone tissues, through gradient elasticity of 1-10, $35-38$, and $62-68 \mathrm{kPa}$. Although soft matrix is able to induce stem cells to neural differentiation, the stiffness is not the same for different matrix materials (Tse and Engler, 2011; Oh et al., 2016). The stiffness of $1-10 \mathrm{kPa}$ was selected in this experiment according to our previous study.

These results suggest that soft matrix regulates neural differentiation of hUC-MSCs, and small molecule inhibition of BMPR can improve neural differentiation of stem cells. Smallmolecule LDN-193189, a selective inhibitor of BMP type I receptors, can selectively suppress the activities of receptorlike kinases (ALK2, ALK3, and ALK6; Han et al., 2015; Chiba et al., 2017). Additionally, BMP ligands can promote the phosphorylation and activation of BMP type I receptors by BMP type II receptors (BMPRII, ActRIIA, and ActRIIB; Miyazono et al., 2010). The activated BMP type I receptors phosphorylate BMP signaling effectors SMAD1/5/8, which regulates gene transcriptions through the transport to the nucleus (Yu et al., 2008). Studies have shown that SMADs localization can be transferred from the nucleus to the cytoplasm when MSCs differentiation is induced on stiff or soft substrates (Du et al., 2011). Therefore, from this study we concluded that BMPR inhibition prevented the entry of SMADs into the nucleus, which further promoted neural differentiation of hUC-MSCs.

Importantly, our results have also proved the synergistic effect of BMPR and integrin $\beta 1$ on soft matrix inducing neural differentiation of hUC-MSCs. It has been demonstrated that integrin $\beta 1$ is the key molecule in the process of cell sensing matrix stiffness (Jiang et al., 2016; Desai et al., 2019). Soft ECM can promote the neural differentiation of BM-MSCs through the endocytosis of integrin $\beta 1$ (Du et al., 2011). In addition, the co-localization of BMPRIA and integrin $\beta 1$ results in receptor internalization, which in turn regulates downstream SMADs phosphorylation and nuclear translocation, thus regulating cell fate (Provenzano et al., 2009; Shih et al., 2011). Based on these data, the observations in this study are consistent with previous reports. In summary, we demonstrated that not only is BMPR involved in the process of MSC differentiation induced by soft matrix, but also there is crosstalk between BMP signaling pathway and integrin-related AKT, GSK-3 $\beta$ signaling. It is reported that MSCs respond to mechanical stimuli through

TABLE 1 | Primer sequences for qRT-PCR.

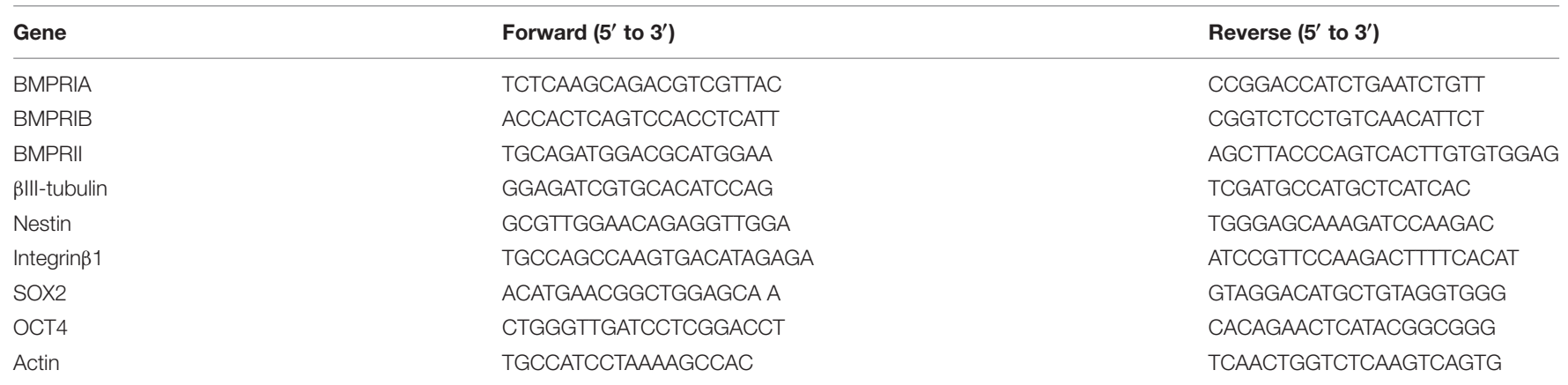


activation of FAK and AKT pathways by vibration induced RhoA signaling, F-actin remodeling, and repression of adipogenic gene expression (Graham and Burridge, 2016). However, further study is needed on how these protein molecules interact with each other.

Understanding how soft surfaces affect molecular signaling pathways could help us to design the materials that strengthen neural differentiation; thus better design surfaces that promote neural function. However, the cells are in a multifactorial internal environment in vivo. Therefore, further researches are needed to be conducted, such as neural differentiation was induced by the combination of soft surfaces and soluble factors, to mimic the ECM environment of cells in vivo, thus promoting later differentiation into specific subtypes of neurons.

\section{MATERIALS AND METHODS}

\section{Cell Culture and Identification}

The culture and identification of hUC-MSCs were based on our previous research methods (Xu et al., 2017). hUC-MSCs were used for all experiments. All experimental procedures were approved by the ethics committee of Jilin University and conformed to the regulatory standards.

\section{Fabrication of Polyacrylamide Matrix With Gradient Stiffness}

Matrixes with gradient stiffness were prepared as previous reports (Engler et al., 2006; Sun et al., 2018). The concentrations of acrylamide (AAm; Sigma-Aldrich, St. Louis, MO, United States) and bis-acrylamide (Bis-AAm; 0.05, 0.3, and 0.7\%; SigmaAldrich) are shown in Figure 1A.

\section{Cell Culture on the Hydrogel and Analysis of Cell Morphology}

Human umbilical cord mesenchymal stem cells were passaged 46 , and then seeded on 6 -well plates $\left(1-1.5 \times 10^{5} / \mathrm{cm}^{2}\right.$ cells $)$ with hydrogel matrix. The growth medium was changed every 3 days. NIH ImageJ was utilized to compute the major and minor axes of the cells based on the binary image of the cells. The aspect ratio of the cell was the ratio of the major to minor axes.

\section{Gene Expression Assay}

Total RNA was isolated with TRI reagent (Takara, Tokyo, Japan), and then a PrimeScript RT reagent kit (Takara) was used to synthesize first strand cDNA. Afterwards, qRT-PCR was applied to examine the relative expression of related genes. The sequences of all primers were shown in Table 1.

\section{Detection of Integrin $\beta 1$ Using Immunofluorescence Staining}

Human umbilical cord mesenchymal stem cells were counted and seeded on a 24-well culture plate with different stiffness at day 1, rinsed with PBS 3 times. The cells were then fixed with $4 \%$ paraformaldehyde for $15 \mathrm{~min}$, and blocked with $1 \%$ bovine serum albumin (BSA) for $1 \mathrm{~h}$. Anti-integrin $\beta 1$ was prepared with
$0.5 \%$ BSA, $300 \mu \mathrm{l}$ was added to each well, and then stored at $4^{\circ} \mathrm{C}$ overnight. After incubation of the secondary antibodies for $1 \mathrm{~h}$, Hoechst33342 (proportion 1:10000) was prepared and used to dye the nucleus for 10-15 min. After rinsing with PBS, the cells were observed under a laser scanning confocal microscope.

\section{Western Blotting}

The proteins were extracted from cells using buffer containing protease and phosphatase inhibitors (Dingguo, Beijing, China). The proteins were separated by electrophoresis on $8 \%$ polyacrylamide gels, and then transferred to polyvinylidene fluoride (PVDF) membranes. Afterwards, the membranes were blocked and probed with antibodies for BMPRIA (Abcam), BMPRIB (Abcam), BMPRII (Abcam), GAPDH (CST), Nestin (CST), $\beta$ II-tubulin (CST), GSK-3 $\beta$ (CST), and FAK (CST) overnight at $4^{\circ} \mathrm{C}$. The secondary antibodies were incubated with the membranes on the next day. Finally, the protein bands were visualized using chemiluminescence.

\section{Statistical Analysis}

Descriptive analysis was performed to describe the characteristics of the variables. Statistical analysis was performed with the Student's unpaired $t$-test and one-way ANOVA. Statistical significance was defined as $p<0.05$. At least three independent experiments were performed in all cases.

\section{DATA AVAILABILITY STATEMENT}

The raw data supporting the conclusions of this article will be made available by the authors, without undue reservation, to any qualified researcher.

\section{ETHICS STATEMENT}

The studies involving human participants were reviewed and approved by the First Hospital of Jilin University Ethics Committee (No. 2019-177). The patients/participants provided their written informed consent to participate in this study.

\section{AUTHOR CONTRIBUTIONS}

YS, LL, and YL were primarily involved in drafting the article. YS performed the experiments and prepared the figures. LL and YL revised the draft manuscript and provided insights. All authors contributed to the writing of the manuscript, and read and approved the final version.

\section{FUNDING}

This work was supported by the National Natural Science Foundation of China (Grant No. 81970547), the Youth Science Foundation of the First Hospital of Jilin University (Grant No. JDYY102019033), and the Promotion and Development plan of Jilin Province (Grant No. 2019Q009). 


\section{REFERENCES}

Al Madhoun, A., Ali, H., Alkandari, S., Atizado, V. L., Akhter, N., Al-Mulla, F., et al. (2016). Defined three-dimensional culture conditions mediate efficient induction of definitive endoderm lineage from human umbilical cord Wharton's jelly mesenchymal stem cells. Stem Cell Res. Ther. 7:165.

Chiba, T., Ishisaki, A., Kyakumoto, S., Shibata, T., Yamada, H., and Kamo, M. (2017). Transforming growth factor-betal suppresses bone morphogenetic protein-2-induced mesenchymal-epithelial transition in HSC-4 human oral squamous cell carcinoma cells via Smad1/5/9 pathway suppression. Oncol. Rep. 37, 713-720. doi: 10.3892/or.2016.5338

Desai, A., Geraghty, S., and Dean, D. (2019). Effects of blocking integrin betal and $\mathrm{N}$-cadherin cellular interactions on mechanical properties of vascular smooth muscle cells. J. Biomech. 82, 337-345. doi: 10.1016/j.jbiomech.2018.11.004

Du, J., Chen, X., Liang, X., Zhang, G., Xu, J., He, L., et al. (2011). Integrin activation and internalization on soft ECM as a mechanism of induction of stem cell differentiation by ECM elasticity. Proc. Natl. Acad. Sci. U.S.A. 108, 9466-9471. doi: 10.1073/pnas.1106467108

Dupont, S., Morsut, L., Aragona, M., Enzo, E., Giulitti, S., Cordenonsi, M., et al. (2011). Role of YAP/TAZ in mechanotransduction. Nature 474, 179-183.

Engler, A. J., Sen, S., Sweeney, H. L., and Discher, D. E. (2006). Matrix elasticity directs stem cell lineage specification. Cell 126, 677-689. doi: 10.1016/j.cell. 2006.06.044

Graham, D. M., and Burridge, K. (2016). Mechanotransduction and nuclear function. Curr.Opin. Cell Biol. 40, 98-105. doi: 10.1016/j.ceb.2016.03.006

Hadden, W. J., Young, J. L., Holle, A. W., Mcfetridge, M. L., Kim, D. Y., Wijesinghe, P., et al. (2017). Stem cell migration and mechanotransduction on linear stiffness gradient hydrogels. Proc. Natl. Acad. Sci. U.S.A. 114, 5647-5652. doi: $10.1073 /$ pnas. 1618239114

Han, K. M., Kim, S. K., Kim, D., Choi, J. Y., Im, I., Hwang, K. S., et al. (2015). Enhanced SMAD1 signaling contributes to impairments of early development in CFC-iPSCs. Stem Cells 33, 1447-1455. doi: 10.1002/stem.1963

Han, Y., Li, X., Zhang, Y., Han, Y., Chang, F., and Ding, J. (2019). Mesenchymal stem cells for regenerative medicine. Cells 8:886.

Her, G. J., Wu, H. C., Chen, M. H., Chen, M. Y., Chang, S. C., and Wang, T. W. (2013). Control of three-dimensional substrate stiffness to manipulate mesenchymal stem cell fate toward neuronal or glial lineages. Acta Biomater. 9, 5170-5180. doi: 10.1016/j.actbio.2012.10.012

Jiang, L., Sun, Z., Chen, X., Li, J., Xu, Y., Zu, Y., et al. (2016). Cells sensing mechanical cues: stiffness influences the lifetime of cell-extracellular matrix interactions by affecting the loading rate. ACS Nanol. 10, 207-217. doi: 10.1021/ acsnano. 5 b03157

Lv, H., Li, L., Sun, M., Zhang, Y., Chen, L., Rong, Y., et al. (2015). Mechanism of regulation of stem cell differentiation by matrix stiffness. Stem Cell Res. Ther. 6:103.

Mao, A. S., Shin, J. W., and Mooney, D. J. (2016). Effects of substrate stiffness and cell-cell contact on mesenchymal stem cell differentiation. Biomaterials 98, 184-191. doi: 10.1016/j.biomaterials.2016.05.004

Miyazono, K., Kamiya, Y., and Morikawa, M. (2010). Bone morphogenetic protein receptors and signal transduction. J. Biochem. 147, 35-51. doi: 10.1093/jb/ mvp148

Oh, S. H., An, D. B., Kim, T. H., and Lee, J. H. (2016). Wide-range stiffness gradient PVA/HA hydrogel to investigate stem cell differentiation behavior. Acta Biomater. 35, 23-31. doi: 10.1016/j.actbio.2016.02.016

Provenzano, P. P., Inman, D. R., Eliceiri, K. W., and Keely, P. J. (2009). Matrix density-induced mechanoregulation of breast cell phenotype, signaling and gene expression through a FAK-ERK linkage. Oncogene 28, 4326-4343. doi: 10.1038/onc.2009.299

Rammensee, S., Kang, M. S., Georgiou, K., Kumar, S., and Schaffer, D. V. (2017). Dynamics of mechanosensitive neural stem cell differentiation. Stem Cells 35, 497-506. doi: 10.1002/stem.2489

Shang, F., Liu, S., Ming, L., Tian, R., Jin, F., Ding, Y., et al. (2017). Human umbilical cord MSCs as new cell sources for promoting periodontal regeneration in inflammatory periodontal defect. Theranostics 7, 4370-4382. doi: 10.7150/thno. 19888

Shih, Y. R., Tseng, K. F., Lai, H. Y., Lin, C. H., and Lee, O. K. (2011). Matrix stiffness regulation of integrin-mediated mechanotransduction during osteogenic differentiation of human mesenchymal stem cells. J. Bone Miner. Res. 26, 730-738. doi: 10.1002/jbmr.278

Smith, L. R., Cho, S., and Discher, D. E. (2018). Stem cell differentiation is regulated by extracellular matrix mechanics. Physiology 33, 16-25. doi: 10.1152/physiol. 00026.2017

Stukel, J. M., and Willits, R. K. (2016). Mechanotransduction of neural cells through cell-substrate interactions. Tissue Eng. Part B Rev. 22, 173-182. doi: 10.1089/ ten.teb.2015.0380

Stukel, J. M., and Willits, R. K. (2018). The interplay of peptide affinity and scaffold stiffness on neuronal differentiation of neural stem cells. Biomed.Mater. 13:024102. doi: 10.1088/1748-605x/aa9a4b

Sun, M., Chi, G., Xu, J., Tan, Y., Xu, J., Lv, S., et al. (2018). Extracellular matrix stiffness controls osteogenic differentiation of mesenchymal stem cells mediated by integrin alpha5. Stem Cell Res. Ther. 9:52.

Sun, Y., Yong, K. M., Villa-Diaz, L. G., Zhang, X., Chen, W., Philson, R., et al. (2014). Hippo/YAP-mediated rigidity-dependent motor neuron differentiation of human pluripotent stem cells. Nat. Mater. 13, 599-604. doi: 10.1038/ nmat3945

Thompson, R., and Chan, C. (2016). Signal transduction of the physical environment in the neural differentiation of stem cells. Technology 4, 1-8. doi: 10.1142/s2339547816400070

Tse, J. R., and Engler, A. J. (2011). Stiffness gradients mimicking in vivo tissue variation regulate mesenchymal stem cell fate. PLoS One 6:e15978. doi: 10.1371/ journal.pone.0015978

Xu, G. K., Yang, C., Du, J., and Feng, X. Q. (2014). Integrin activation and internalization mediated by extracellular matrix elasticity: a biomechanical model. J. Biomech. 47, 1479-1484. doi: 10.1016/j.jbiomech.2014. 01.022

Xu, J., Sun, M., Tan, Y., Wang, H., Wang, H., Li, P., et al. (2017). Effect of matrix stiffness on the proliferation and differentiation of umbilical cord mesenchymal stem cells. Differentiation 96, 30-39. doi: 10.1016/j.diff.2017.07.001

Yu, P. B., Deng, D. Y., Lai, C. S., Hong, C. C., Cuny, G. D., Bouxsein, M. L., et al. (2008). BMP type I receptor inhibition reduces heterotopic [corrected] ossification. Nat. Med. 14, 1363-1369. doi: 10.1038/nm.1888

Conflict of Interest: The authors declare that the research was conducted in the absence of any commercial or financial relationships that could be construed as a potential conflict of interest.

Copyright (C) 2020 Sun, Xu, Wang, Lv, Wu, Chi, Li and Li. This is an open-access article distributed under the terms of the Creative Commons Attribution License (CC BY). The use, distribution or reproduction in other forums is permitted, provided the original author(s) and the copyright owner(s) are credited and that the original publication in this journal is cited, in accordance with accepted academic practice. No use, distribution or reproduction is permitted which does not comply with these terms. 